\title{
ORIGINAL
}

\section{PATRONES DE CONSUMO DE ALCOHOL EN ESPAÑA: UN PAÍS EN TRANSICIÓN}

\author{
Iñaki Galán (1,2), Mª José González (3) y José L Valencia-Martín $(2,4)$.
}

(1) Centro Nacional de Epidemiología. Instituto de Salud Carlos III. Madrid.

(2) Departamento de Medicina Preventiva y Salud Pública. Universidad Autónoma de Madrid/IdiPAZ

(3) Departamento de Evaluación y Calidad. Madrid Salud. Ayuntamiento de Madrid

(4) Servicio de Medicina Preventiva. Hospital Universitario de Móstoles.

Los autores declaran no tener conflicto de intereses.

\section{RESUMEN}

Fundamentos: El modo en el que se consume alcohol en los países mediterráneos se encuentra en proceso de transición. El objetivo de este trabajo es describir los patrones de consumo en la población adulta española según las principales características sociodemográficas.

Métodos: Estudio transversal con participantes de 15 años y más utlizando como fuente de información la Encuesta Nacional de Salud (ENS) 2011-2012 ( $\mathrm{n}=20.608)$. Según la ingesta promedio de alcohol, se clasificó como de alto riesgo el consumo $\geq 40 \mathrm{~g} /$ día de alcohol en hombres o $\geq 24$ $\mathrm{g}$ /día en mujeres. Se definió binge drinking el consumo de $\geq 6$ bebidas estándar de alcohol (hombres) y $>5$ (mujeres) en 4-6 horas durante los últimos 12 meses. Se estimó la preferencia de bebida y la tendencia 1987 2012 (ENS respectivas). Se analizó su distribución con variables sociodemográficas mediante modelos ajustados de regresión logística.

Resultados: El 1,3\% de los sujetos encuestados fueron bebedores promedio de alto riesgo ( $2 \%$ de hombres y $0,7 \%$ de mujeres). En hombres fue más frecuente entre los 45-64 años (odds ratio $(\mathrm{OR})=2,92$ respecto a 15-29 años; IC 95\%:1,59-5,38) y en los sujetos nacidos en España $(\mathrm{OR}=3,45$; IC 95\%: 1,59-7,69). En mujeres se observaron diferencias estadísticamente significativas según el nivel educativo, incrementándose a medida que aumentaba éste ( $\mathrm{p}$ tendencia lineal $<0,001$ ). El 19,6\% de hombres y $7,1 \%$ y de mujeres realizaron binge drinking en el último año. Este patrón disminuyó con la edad y se incrementó con el nivel de estudios en ambos sexos ( $p$ tendencia lineal $<0,001$ ). La prevalencia estandarizada de bebedores promedio de alto riesgo descendió desde un $18,8 \%$ en 1987 hasta el 1,3\% en 2012 .

Conclusiones: El consumo de alcohol en España es compatible con el modelo de países en transición, donde el binge drinking es el que más contribuye al riesgo global. Se observa un importante descenso del consumo promedio de alto riesgo. La cerveza desplaza al vino como bebida preferente.

Palabras clave: Consumo de bebidas alcohólicas. Vino. Cerveza Licores. Epidemiología. España.

\section{Correspondencia}

Iñaki Galán

Centro Nacional de Epidemiología

Instituto de Salud Carlos III

Monforte de Lemos, 5

28029 Madrid

igalan@isciii.es

\section{ABSTRACT}

\section{Alcohol Drinking Patterns in Spain: a Country in Transition}

Background: Alcohol consumption in Mediterranean countries is in a transition period. The objective is to describe in the Spanish adult population the pattern of alcohol consumption by major sociodemographic variables.

Methods: A cross-sectional study among 20,608 individuals aged $\geq 15$ years who participated in the Spanish Health Interview Survey (ENS) 2011-2012. According to average intake, people were classified as heavy drinkers if they drank $\geq 40 \mathrm{~g} /$ day of alcohol (men) or $\geq 24 \mathrm{~g} /$ day (women). Binge drinking was defined as the consumption of $\geq 6$ standard drinks (men) and $\geq 5$ (women) at any drinking occasion (4-6 hours) in the last year. The beverage preference and the trend for 1987-2012 (ENS of these years) were estimated. The relationship of the distribution of drinking patterns with sociodemographic variables was analyzed using adjusted logistic regression models.

Results: The prevalence of heavy drinkers was $1.3 \%$ (2\% in men and $0.7 \%$ in women). In men, heavy drinking was more frequent among 45 to 64 year olds (odds ratio $(\mathrm{OR})=2.92$ compared to men of $15-29$ years; CI $95 \%: 1.59-5.38)$ and those born in Spain ( $\mathrm{OR}=3.45$; CI 95\%: 1.59-7.69). In women, the only differences observed were those regarding education level, with heavy drinking increasing as the level of education increases ( $p$ linear trend $<0.001)$. The prevalence of binge drinking during the last year was $19.6 \%$ in men and $7.1 \%$ in women. In both genders, the risk of binge drinking decreased with age and increased with higher education level (p linear trend $<0.001$ ). The standardized prevalence of heavy drinkers has declined from $18.8 \%$ in 1987 to $1.3 \%$ in 2012.

Conclusion: Alcohol consumption in Spain is consistent with the model for countries in transition, where binge drinking is the most common pattern of excessive use of alcohol. A strong decline in heavy drinkers was observed and wine has been displaced by beer in beverage preference.

Keyword: Alcohol drinking. Wine. Beer. Spirits. Epidemiology. Binge drinking. Spain. 


\section{INTRODUCCIÓN}

El consumo de alcohol es responsable del $3,8 \%$ de la mortalidad general y la tercera causa de mortalidad prematura y discapacidad, contribuyendo a un $4,5 \%$ del total de años de vida ajustados por discapacidad (DALYs) ${ }^{1}$.

Existen diferentes patrones de consumo de alcohol. Tradicionalmente se ha dividido el mundo en regiones con elevado o bajo consumo per cápita, denominando wet cultures o dry cultures respectivamente. En países con wet cultures el consumo de alcohol está integrado en la vida cotidiana y es consumido regularmente acompañando las comidas, las bebidas alcohólicas son fácilmente accesibles y la proporción de personas abstemias es escasa. Esta cultura es característica de los países de la cuenca mediterránea en los que el vino es la bebida predominante, a diferencia de las regiones con dry cultures donde el consumo de alcohol no es habitual con las comidas ni está integrado en las actividades cotidianas. Su acceso es más restrictivo y las tasa de abstinencia son muy superiores. Sin embargo, cuando se consume alcohol es más probable que ocurra intoxicación. Este patrón de consumo es típico de los países escandinavos, Estados Unidos y Canadá, donde la cerveza y los destilados son las bebidas preferentes. Comparaciones más recientes realizadas principalmente en países europeos, describen como la división wet/dry parece ir desapareciendo y la forma de consumo, incluido el tipo de bebidas, se va homogeneizan$\mathrm{do}^{2}$. Por otro lado, esta dicotomía se ha adaptado a nuevas formas de consumo emergentes, considerando una variedad de comportamientos donde destacan las dimensiones de la regularidad del consumo y del grado de embriaguez ${ }^{3}$.

España podría situarse en este perfil de países en transición. A pesar de que el patrón de consumo es menos peligroso comparado con el de otros países ${ }^{4}$, el por- centaje de personas que realizan consumos excesivos en una misma ocasión o binge drinking en población adulta es elevado $^{5,6}$ y el número de intoxicaciones etílicas continúa aumentando en la población juvenil ${ }^{7}$. Probablemente, de seguir este cambio de patrón, las consecuencias del consumo de alcohol podrían incrementarse en un futuro ${ }^{8}$.

Desde una perspectiva de salud pública, a pesar de que el estudio del consumo de alcohol es extraordinariamente complejo, disponer de una aproximación cuantitativa del nivel de consumo, su distribución entre los diferentes estratos poblacionales y su tendencia, es una de las principales tareas para planificar las diferentes estrategias de intervención ${ }^{9}$.

En España todavía no están adecuadamente descritos los diferentes patrones de consumo, a pesar de que la Encuesta Nacional de Salud ${ }^{10}$ y la Encuesta Domiciliaria de Drogas $^{11}$ incorporan un módulo para estimar el consumo de alcohol.

El objetivo de este estudio es describir los patrones de consumo de alcohol teniendo en cuenta el consumo promedio, el binge drinking y la bebida preferida, según las principales características sociodemográficas.

\section{MATERIAL Y MÉTODOS}

Estudio transversal basado en los datos de la Encuesta Nacional de Salud (ENS) 2011-2012 $2^{10}$. La población de estudio fue el conjunto de personas de 15 o más años que residía en viviendas familiares principales de todo el territorio nacional. Se seleccionó a las personas participantes en la muestra a través de un procedimiento multietápico estratificado, correspondiendo los estratos a municipios ordenados según tamaño. La recogida de información se realizó desde julio de 2011 hasta junio de 2012 mediante entrevista personal asistida por ordenador (CAPI). 
La tasa de respuesta, definida como el porcentaje de hogares encuestados en las viviendas titulares, fue del $71,1 \%$. La falta de respuesta estuvo originada en el 13,7\% en ausencias, el $14,6 \%$ en negativas a participar y el $0,7 \%$ en incapacidad para contes$\operatorname{tar}^{12}$. El tamaño muestral fue de 21.007 personas de 15 o más años. A efectos de este trabajo, se excluyeron 399 individuos en los que no se pudo obtener información de la ingesta de bebidas alcohólicas o del patrón binge drinking.

Tendencias: para el estudio de tendencias se utilizaron los datos de las Encuestas Nacionales de Salud (ENS) de 1987, 1993, 1995, 1997, 2001, 2006, fracción para España de la Encuesta Europea de Salud 2009 y ENS 2011-2012. No se utilizaron los datos de la ENS de 2003 debido a las diferencias metodológicas que impedían la comparación de las estimaciones.

Variables:Magnitud y distribución actual. En la ENS 2011-2012 la ingesta de alcohol se estimó a partir de la frecuencia realizada a lo largo de una semana tipo en el último año, diferenciando para cada día de la semana la cantidad consumida de 6 tipos de bebidas: cerveza, vinos-cava, bebidas "locales", vermut-fino-jerez, licores-anís-pacharán, whisky-coñac-combinados. Para realizar la conversión a gramos de alcohol puro, se le asignaron 9,09, 10,09, 19,82 g de alcohol correspondientes a una unidad de bebida estándar a la cerveza, vino y licores de alta graduación, según el estudio de Llopis et al. ${ }^{13} \mathrm{y}$ al resto de bebidas se les asignó $10 \mathrm{~g}$.

Se estimó la prevalencia de consumo de alcohol a lo largo de la vida, durante el último año y de bebedores diarios, así como la de exbebedores o bebedores anteriores (no consumidores durante el último año pero sí previamente).

Según el consumo promedio se clasificó en no bebedores actuales a los que no consumieron alcohol en los últimos 12 meses, bebedor de bajo riesgo si consumieron entre 1-39 g/día en hombres y 1-23 g/día en mujeres, $\mathrm{y}$ bebedores de alto riesgo si consumieron $\geq 40 \mathrm{~g} /$ día (hombres) $\mathrm{o} \geq 24$ $\mathrm{g} /$ día (mujeres) ${ }^{14}$.

El binge drinking se define como el consumo de $\geq 6$ bebidas estándar en un intervalo de cuatro a seis horas en el caso de los hombres y $\geq 5$ en las mujeres, en los últimos 12 meses (una bebida estándar contiene $10 \mathrm{~g}$ de alcohol).

Se clasificó el consumo de alcohol en cinco tipos combinando el consumo promedio y el patrón binge drinking (no bebedores, bebedores con consumo promedio de bajo riesgo con o sin binge drinking y bebedores con consumo promedio de alto riesgo con o sin binge drinking).

Se estimó la preferencia de consumo de vino, cerveza y licores de alta graduación cuando la ingesta de estas bebidas en las personas que realizaron un consumo de alcohol de al menos dos veces al mes, supuso el $80 \%$ o más del total de la ingesta de alcohol. Se clasificó "sin preferencia" cuando estas bebidas no alcanzaron el $80 \%{ }^{15}$.

Para clasificar el consumo promedio de alcohol, las ENS 1987, 1993, 1995, 1997, 2001, 2006 y la fracción para España de la Encuesta Europea de Salud 2009, utilizaron un cuestionario de frecuencia de consumo habitual con mínimas modificaciones del tipo de bebidas. Una excepción fue el año 2003 que no permitía cuantificar la ingesta total de alcohol por cómo estaban formuladas las preguntas, motivo por el que fue excluido del análisis. Para realizar la conversión se utilizaron los mismos contenidos de alcohol descritos previamente.

Análisis estadístico: se calculó la prevalencia de consumo y se estimaron mediante regresión logística multinomial los Relative Risk Ratios [los interpretaremos como odds ratios (OR)] del consumo de bajo riesgo y 
de alto riesgo por sexo, edad, nivel de estudios y país de nacimiento. De igual forma se elaboraron modelos de regresión logística binaria para el binge drinking. Se estimó la $\mathrm{p}$ de tendencia lineal de prevalencia según la edad y nivel de estudios. Para analizar las tendencias de consumo promedio de alto riesgo se ajustaron por edad las prevalencias de bebedores, utilizando como población estándar la media para todo el periodo.

Se evaluó la existencia de interacciones entre las variables sociodemográficas estudiadas. Dado que se observaron interacciones estadísticamente significativas entre el sexo y otras variables de estudio, los análisis se realizaron de forma desagregada para hombres y mujeres.

Todos los análisis fueron realizados con Stata v12 para Windows (1984-2013 StataCorp, Texas, USA).

\section{RESULTADOS}

De forma general, para el período 20112012 , el $77,5 \%$ de la población española había consumido alcohol a lo largo de la vida, el $65,4 \%$ lo consumió en el último año y el $13,8 \%$ de forma diaria. El 12,1\% eran exbebedores o bebedores anteriores, es decir, habían consumido alcohol previamente pero no durante el ultimo año.

En la tabla 1 se describe la clasificación según el consumo promedio de alcohol y la distribución de la prevalencia según las variables sociodemográficas. La proporción de no bebedores actuales (no consumo en los últimos 12 meses) fue del $22,6 \%$ en los hombres y del $46 \%$ en las mujeres. La categoría más frecuente en ambos sexos fue la de bebedores de bajo riesgo, un 75,4\% de los hombres y $53,4 \%$ de las mujeres. Finalmente, el 2,0\% (IC 95\%: 1,7-2,3) de los hombres y el 0,7\% (IC 95\%: 0,5-0,9) de las mujeres eran bebedores de alto riesgo desde la perspectiva del consumo promedio.
En la tabla 2 se analiza la distribución de la probabilidad de consumo promedio de bajo riesgo y de alto riesgo comparado con la categoría de no bebedores, ajustando por todas las variables sociodemográficas simultáneamente. El consumo de bajo riesgo en los hombres fue más frecuente entre $\operatorname{los} 30$ y 64 años, aumentaba a medida que se incrementaba el nivel educativo ( $p$ tendencia lineal $<0,001)$ y era menor en los sujetos nacidos en el extranjero. En las mujeres, la distribución por nivel de estudios y país de nacimiento siguió el mismo patrón que en los hombres. Sin embargo, el consumo de bajo riesgo se realizó con mayor frecuencia en el grupo más joven. Respecto al consumo promedio de alto riesgo, los hombres de 4564 años mostraron un OR de 2,92 (IC 95\%: $1,59-5,38)$ respecto a los más jóvenes. No se observaron diferencias con el nivel de estudios pero sí con el país de nacimiento, donde las personas nacidas fuera de España tenían menor probabilidad de ser bebedoras de alto riesgo (OR: 0,29; IC 95\%: 0,13$0,63)$. En las mujeres no se observaron diferencias en la edad o país de nacimiento pero sí con el nivel de estudios, ya que la probabilidad de realizar un consumo de riesgo aumentó con el nivel educativo ( $\mathrm{p}$ tendencia lineal $<0,001$ ), siendo máxima en las mujeres universitarias frente a las que tenían estudios primarios o inferiores (OR: 11,92; IC 95\%: 3,23-43,97).

En la tabla 3 se puede observar la magnitud y distribución del binge drinking. El 19,6\% (IC 95\%: 18,8-20,8) de los hombres y el 7,1\% (IC 95\%: 6,7-8,0) de las mujeres realizaron este consumo en el último año, mientras que el $7,1 \%$ y $2,1 \%$ de hombres y mujeres tuvo este patrón mensualmente. Su distribución fue bastante similar entre hombres y mujeres: en ambos disminuyó a medida que aumentaba la edad y se incrementó al aumentar el nivel de estudios ( $p$ tendencia lineal $<0,001$ ), especialmente en las mujeres, en las que se observó un OR de 3,63 (IC 95\%: 2,37-5,58) en el grupo de universitarias en relación a las que tenían 
Tabla 1

Consumo promedio de alcohol según características sociodemográficas en la población $\geq 15$ años. Encuesta Nacional de Salud 2011-2012

\begin{tabular}{|c|c|c|c|c|c|c|c|c|c|}
\hline & \multicolumn{3}{|c|}{ No bebedor* } & \multicolumn{3}{|c|}{ Bebedor de bajo riesgo $^{\dagger}$} & \multicolumn{3}{|c|}{ Bebedor de alto riesgo } \\
\hline & $\begin{array}{c}\text { Total } \\
n=7.134\end{array}$ & $\begin{array}{l}\text { Hombre } \\
n=2.270\end{array}$ & $\begin{array}{c}\text { Mujer } \\
n=4.864\end{array}$ & \begin{tabular}{|c|} 
Total \\
$\mathrm{n}=13.204$
\end{tabular} & $\begin{array}{l}\text { Hombre } \\
n=7.559\end{array}$ & $\begin{array}{c}\text { Mujer } \\
n=5.645\end{array}$ & $\begin{array}{c}\text { Total } \\
n=270\end{array}$ & $\begin{array}{c}\text { Hombre } \\
n=198\end{array}$ & $\begin{array}{l}\text { Mujer } \\
\mathrm{n}=72\end{array}$ \\
\hline & $\%$ & $\%$ & $\%$ & $\%$ & $\%$ & $\%$ & $\%$ & $\%$ & $\%$ \\
\hline Total & 34,6 & 22,6 & 46,0 & 64,1 & 75,4 & 53,4 & 1,3 & 2,0 & 0,7 \\
\hline \multicolumn{10}{|l|}{ Edad } \\
\hline $15-29$ & 29,7 & 24,0 & 35,8 & 69,4 & 74,9 & 63,5 & 0,9 & 1,1 & 0,7 \\
\hline $30-44$ & 28,9 & 18,8 & 39,2 & 70,3 & 80,2 & 60,2 & 0,7 & 1,0 & 0,5 \\
\hline $45-64$ & 31,9 & 19,8 & 43,5 & 66,2 & 77,2 & 55,6 & 1,9 & 3,0 & 0,9 \\
\hline$\geq 65$ & 51,7 & 32,6 & 66,0 & 46,7 & 64,5 & 33,5 & 1,6 & 3,0 & 0,6 \\
\hline Valor de $\mathrm{p}$ & $<0,001$ & $<0,001$ & $<0,001$ & $<0,001$ & $<0,001$ & $<0,001$ & $<0,001$ & $<0,001$ & 0,519 \\
\hline \multicolumn{10}{|l|}{ Nivel de estudios } \\
\hline Primarios o menos & 52,9 & 36,4 & 66,1 & 45,4 & 60,1 & 33,7 & 1,6 & 3,5 & 0,2 \\
\hline E. Secundarios $1^{\mathrm{a}}$ etapa & 34,8 & 22,8 & 47,4 & 63,6 & 75,2 & 51,6 & 1,6 & 2,0 & 1,0 \\
\hline E. Secundarios $2^{\mathrm{a}}$ etapa & 26,5 & 16,5 & 36,7 & 72,6 & 82,4 & 62,7 & 0,9 & 1,1 & 0,7 \\
\hline E. Universitarios & 22,4 & 15,5 & 28,5 & 76,5 & 83,1 & 70,7 & 1,1 & 1,4 & 0,8 \\
\hline Valor de $\mathrm{p}$ & $<0,001$ & $<0,001$ & $<0,001$ & $<0,001$ & $<0,001$ & $<0,001$ & 0,005 & $<0,001$ & 0,010 \\
\hline \multicolumn{10}{|l|}{ País de nacimiento } \\
\hline España & 33,9 & 21,2 & 46,1 & 64,7 & 76,7 & 53,3 & 1,4 & 2,1 & 0,7 \\
\hline Extranjero & 39,0 & 31,7 & 45,5 & 60,2 & 67,4 & 53,7 & 0,8 & 0,8 & 0,8 \\
\hline Valor de $\mathrm{p}$ & $<0,001$ & $<0,001$ & 0,788 & 0,002 & $<0,001$ & 0,843 & 0,062 & 0,008 & 0,655 \\
\hline
\end{tabular}

* No bebedor: no ha consumido alcohol en los últimos 12 meses. ‘Bebedor de bajo riesgo: ha consumido alcohol en los últimos 12 meses con cualquier frecuencia y cantidad $<40 \mathrm{~g} /$ día en hombres y $<24 \mathrm{~g} /$ día en mujeres. ${ }^{\ddagger}$ Bebedor de alto riesgo: ha consumido alcohol en los últimos 12 meses con cualquier frecuencia y cantidad $\geq 40 \mathrm{~g} /$ día en hombres $\mathrm{y} \geq 24$ $\mathrm{g} /$ día en mujeres

estudios primarios o inferiores. Las personas nacidas en el extranjero tuvieron menor probabilidad de realizar este patrón aunque las diferencias ajustadas sólo fueron estadísticamente significativas en los hombres (OR: 0,80; IC 95\%: 0,64-0,99).

En la tabla 4 se describen los tipos de consumo de alcohol combinando la ingesta promedio y el patrón binge drinking, destacando que el $12,4 \%$ del total de individuos eran consumidores promedio de bajo riesgo con patrón binge drinking, lo que representó que el $94 \%$ de las personas que realizaron binge drinking estuvieron clasificadas como consumidores de bajo riesgo a partir de la ingesta promedio. El 0,8\% tenían el máximo riesgo al confluir los dos patrones.
Como se puede apreciar en la tabla 5, globalmente la cerveza fue la bebida preferida, para el $28,7 \%$ de las personas la mayor parte de la ingesta de alcohol puro fue de esta bebida, ligeramente por encima del vino $(23 \%)$. En las mujeres, sin embargo, el consumo de ambas fue muy similar. En ambos sexos la preferencia del vino aumentó con la edad, mientras que ocurrió lo contrario con los licores de alta graduación que, junto con la cerveza, fueron las bebidas preferidas en el grupo más joven.

Finalmente, en la tabla 6 podemos observar la evolución del consumo promedio de alto riesgo desde 1987 hasta la actualidad. Globalmente, de una prevalencia estandarizada en 1987 del 18,8\%, descendió hasta el 
Tabla 2

Distribución del consumo promedio de alcohol de bajo y alto riesgo según características sociodemográficas en bebedores $\geq 15$ años. Encuesta Nacional de Salud 2011-2012

\begin{tabular}{|c|c|c|c|c|c|c|c|c|}
\hline & \multicolumn{4}{|c|}{ HOMBRES } & \multicolumn{4}{|c|}{ MUJERES } \\
\hline & \multicolumn{2}{|c|}{$\begin{array}{c}\text { Bebedor } \\
\text { de bajo riesgo* }\end{array}$} & \multicolumn{2}{|c|}{$\begin{array}{c}\text { Bebedor } \\
\text { de alto riesgo }{ }^{\dagger}\end{array}$} & \multicolumn{2}{|c|}{$\begin{array}{c}\text { Bebedoras } \\
\text { de bajo riesgo }\end{array}$} & \multicolumn{2}{|c|}{$\begin{array}{c}\text { Bebedoras } \\
\text { de alto riesgo }\end{array}$} \\
\hline & $\mathrm{OR}^{\ddagger}$ & IC $95 \%$ & $\mathrm{OR}^{\ddagger}$ & IC $95 \%$ & $\mathrm{OR}^{\ddagger}$ & IC 95\% & $\mathrm{OR}^{\ddagger}$ & IC 95\% \\
\hline \multicolumn{9}{|l|}{ Edad } \\
\hline $15-29$ & 1 & & 1 & & 1 & & 1 & \\
\hline $30-44$ & 1,26 & $1,04-1,53$ & 1,14 & $0,58-2,24$ & 0,80 & $0,68-0,95$ & 0,66 & $0,26-1,62$ \\
\hline $45-64$ & 1,21 & $1,00-1,47$ & 2,92 & $1,59-5,38$ & 0,80 & $0,68-0,94$ & 1,30 & $0,54-3,09$ \\
\hline$\geq 65$ & 0,80 & $0,65-0,98$ & 1,61 & $0,79-3,28$ & 0,43 & $0,36-0,52$ & 1,05 & $0,31-3,60$ \\
\hline $\mathrm{p}$ tendencia lineal & 0,008 & & 0,016 & & $<0,001$ & & 0,948 & \\
\hline \multicolumn{9}{|l|}{ Nivel de estudios } \\
\hline Primarios o menos & 1 & & 1 & & 1 & & 1 & \\
\hline E. Secundarios $1^{\mathrm{a}}$ etapa & 1,84 & $1,56-2,18$ & 1,06 & $0,67-1,68$ & 1,63 & $1,42-1,87$ & 8,71 & $2,41-31,42$ \\
\hline E. Secundarios $2^{\mathrm{a}}$ etapa & 2,78 & $2,30-3,36$ & 0,85 & $0,50-1,44$ & 2,44 & $2,09-2,85$ & 7,71 & $2,23-26,71$ \\
\hline E. Universitarios & 2,88 & $2,30-3,59$ & 1,03 & $0,55-1,92$ & 3,52 & $2,94-4,22$ & 11,92 & $3,23-43,97$ \\
\hline $\mathrm{p}$ tendencia lineal & $<0,001$ & & 0,753 & & $<0,001$ & & $<0,001$ & \\
\hline \multicolumn{9}{|l|}{ País de nacimiento } \\
\hline España & 1 & & 1 & & 1 & & 1 & \\
\hline Extranjero & 0,50 & $0,41-0,61$ & 0,29 & $0,13-0,63$ & 0,78 & $0,66-0,93$ & 1,08 & $0,42-2,77$ \\
\hline
\end{tabular}

*Bebedor de bajo riesgo: Ha consumido alcohol en los últimos 12 meses con cualquier frecuencia y cantidad $<40 \mathrm{~g} /$ día en hombres y $<24 \mathrm{~g} /$ día en mujeres. "Bebedor de alto riesgo: Ha consumido alcohol en los últimos 12 meses con cualquier frecuencia y cantidad $\geq 40 \mathrm{~g} /$ día en hombres y $\geq 24 \mathrm{~g}$ /día en mujeres. ${ }^{\ddagger}$ Odds ratios estimados a través de regresión logística multinomial, utilizando a los no bebedores como categoría de referencia y ajustando por todas las variables simultáneamente

\section{Tabla 3}

Distribución del patrón binge drinking* según características sociodemográficas en la población $\geq 15$ años. Encuesta Nacional de Salud 2011-2012

\begin{tabular}{|c|c|c|c|c|c|c|}
\hline & \multicolumn{4}{|c|}{ Hombres $n=10.026$} & \multicolumn{2}{|c|}{ Mujeres $n=10.582$} \\
\hline & $\%$ & $\mathrm{OR}^{\dagger}$ & IC 95\% & $\%$ & $\mathrm{OR}^{\dagger}$ & IC 95\% \\
\hline Total & 19,6 & & & 7,1 & & \\
\hline \multicolumn{7}{|l|}{ Edad } \\
\hline $15-29$ & 31,6 & 1 & & 15,2 & 1 & \\
\hline $30-44$ & 24,3 & 0,68 & $0,57-0,81$ & 9,5 & 0,56 & $0,44-0,70$ \\
\hline $45-64$ & 15,1 & 0,38 & $0,32-0,46$ & 4,6 & 0,30 & $0,23-0,39$ \\
\hline$\geq 65$ & 5,7 & 0,14 & $0,11-0,19$ & 1,0 & 0,10 & $0,06-0,16$ \\
\hline $\mathrm{p}$ tendencia lineal & $<0,001$ & & & $<0,001$ & & \\
\hline \multicolumn{7}{|l|}{ Nivel de estudios } \\
\hline Primarios o menos & 11,1 & 1 & & 1,6 & 1 & \\
\hline E. Secundarios $1^{\mathrm{a}}$ etapa & 20,5 & 1,28 & $1,04-1,59$ & 6,4 & 2,21 & $1,45-3,37$ \\
\hline E. Secundarios $2^{\mathrm{a}}$ etapa & 23,7 & 1,49 & $1,21-1,85$ & 10,6 & 3,29 & $2,17-5,01$ \\
\hline E. Universitarios & 21,4 & 1,39 & $1,09-1,78$ & 11,3 & 3,63 & $2,37-5,58$ \\
\hline $\mathrm{p}$ tendencia lineal & $<0,001$ & & & $<0,001$ & & \\
\hline \multicolumn{7}{|l|}{ País de nacimiento } \\
\hline España & 19,6 & 1 & & 7,0 & 1 & \\
\hline Extranjero & 20,1 & 0,80 & $0,64-0,99$ & 8,1 & 0,85 & $0,62-1,15$ \\
\hline Valor de $\mathrm{p}$ & 0,776 & & & 0,307 & & \\
\hline
\end{tabular}

* Binge drinking: consumo de $\geq 6$ bebidas estándar ( $10 \mathrm{~g}$ de alcohol) en un intervalo de cuatro a seis horas en el caso de los hombres y $\geq 5$ en las mujeres. 'Odds ratios estimados a través de regresión logística binaria y ajustando por todas las variables simultáneamente 
Tabla 4

Tipos de consumo de alcohol según edad y sexo

en la población $\geq 15$ años. Encuesta Nacional de Salud 2011-2012

\begin{tabular}{|l|c|c|c|c|c|}
\hline & $\begin{array}{c}\text { No bebedor } \\
\mathrm{n}=7.134\end{array}$ & $\begin{array}{c}\text { PBR-No-BD } \\
\mathrm{n}=10.653\end{array}$ & $\begin{array}{c}\text { PBR-Sí-BD } \\
\mathrm{n}=2.551\end{array}$ & $\begin{array}{c}\text { PAR-No-BD } \\
\mathrm{n}=96\end{array}$ & $\begin{array}{c}\text { PAR-Sí-BD } \\
\mathrm{n}=174\end{array}$ \\
\cline { 2 - 6 } & $\%$ & $\%$ & $\%$ & 0,5 & 0,8 \\
\hline Total & 34,6 & 51,7 & 12,4 & 0,7 & 1,3 \\
\hline Hombres & 22,6 & 57,1 & 18,3 & 0,0 & 1,1 \\
\hline Edad & & & & 0,1 & 0,8 \\
\hline $15-29$ & 24,0 & 44,5 & 30,4 & 0,9 & 2,0 \\
\hline $30-44$ & 18,8 & 56,7 & 23,5 & 1,9 & 1,0 \\
\hline $45-64$ & 19,8 & 64,2 & 13,0 & $<0,001$ & 0,002 \\
\hline$\geq 65$ & 32,6 & 59,8 & 4,6 & 0,3 & 0,4 \\
\hline $\mathrm{p}$ & $<0,001$ & $<0,001$ & $<0,001$ & & 0,7 \\
\hline Mujeres & 46,0 & 46,7 & 6,7 & 0,0 & 0,4 \\
\hline Edad & & & & 0,1 & 0,5 \\
\hline $15-29$ & 35,8 & 49,0 & 14,5 & 0,4 & 0,1 \\
\hline $30-44$ & 39,3 & 51,1 & 9,2 & 0,5 & 0,090 \\
\hline $45-64$ & 43,5 & 51,5 & 4,2 & 0,090 & 0,9 \\
\hline$\geq 65$ & 66,0 & 32,6 & $<0,001$ & & 0 \\
\hline $\mathrm{p}$ & $<0,001$ & $<0,001$ & & & \\
\hline
\end{tabular}

No bebedor: No ha consumido alcohol en los últimos 12 meses; PBR: bebedor con consumo promedio de bajo riesgo (ha consumido alcohol en los últimos 12 meses con cualquier frecuencia y cantidad $<40 \mathrm{~g} /$ día en hombres y $<24 \mathrm{~g} /$ día en mujeres); PAR: bebedor con consumo promedio de alto riesgo (ha consumido alcohol en los últimos 12 meses con cualquier frecuencia y cantidad $\geq 40 \mathrm{~g} /$ día en hombres y $\geq 24 \mathrm{~g}$ /día en mujeres); BD: Binge drinking Sí/No (consumo de $\geq 6$ bebidas estándar ( $10 \mathrm{~g}$ de alcohol) en un intervalo de 4-6 horas en los hombres y $\geq 5$ en las mujeres en los últimos 12 meses)

Tabla 5

Preferencia de bebida*según edad y sexo en la población $\geq 15$ años. Encuesta Nacional de Salud 2011-2012

\begin{tabular}{|l|c|c|c|c|}
\hline & Sin preferencia & Vino & Cerveza & Licores alta graduación \\
\hline & $\%$ & $\%$ & $\%$ & $\%$ \\
\hline Total $\left(\mathrm{n}=9.492^{\dagger}\right)$ & 39,8 & 23,0 & 28,7 & 7,8 \\
\hline Hombres $\left(\mathrm{n}=6.098^{\dagger}\right)$ & 43,9 & 19,7 & 28,5 & 7,3 \\
\hline Edad & & & & 22,9 \\
\hline $15-29$ & 49,9 & 2,1 & 24,9 & 5,2 \\
\hline $30-44$ & 48,1 & 8,0 & 38,0 & 3,9 \\
\hline $45-64$ & 43,3 & 23,2 & 29,2 & 1,7 \\
\hline$\geq 65$ & 30,2 & 54,1 & 12,6 & $<0,001$ \\
\hline Valor de $\mathrm{p}$ & $<0,001$ & $<0,001$ & $<0,001$ & 8,7 \\
\hline Mujeres $\left(\mathrm{n}=3.394^{\dagger}\right)$ & 32,4 & 28,9 & 29,1 & 33,6 \\
\hline Edad & & & & 3,9 \\
\hline $15-29$ & 37,5 & 4,6 & 23,1 & 1,3 \\
\hline $30-44$ & 37,8 & 18,6 & 38,8 & 2,4 \\
\hline $45-64$ & 31,9 & 36,2 & 29,7 & $<0,001$ \\
\hline$\geq 65$ & 16,0 & 64,2 & 16,8 & \\
\hline Valor de $\mathrm{p}$ & $<0,001$ & $<0,001$ & $<0,001$ & \\
\hline
\end{tabular}

* Las bebidas que contribuyen a un $80 \%$ del total de la ingesta de alcohol. "Individuos que consumen alcohol al menos dos veces al mes. 
Tabla 6

Evolución de la prevalencia de bebedores promedio de alto riesgo* en la población $\geq 15$ años.

Encuesta Nacional de Salud, 1987-2012

\begin{tabular}{|l|c|c|c|c|c|c|}
\hline & \multicolumn{2}{|c|}{ TOTAL } & \multicolumn{2}{c|}{ HOMBRES } & \multicolumn{2}{c|}{ MUJERES } \\
\hline & $\% \dagger$ & IC $95 \%$ & $\% \dagger$ & IC 95\% & $\% \dagger$ & IC 95\% \\
\hline 1987 & 18,8 & $18,3-19,2$ & 28,7 & $27,9-29,5$ & 9,8 & $9,3-10,3$ \\
\hline 1993 & 12,0 & $11,6-12,5$ & 21,2 & $20,3-22,0$ & 3,9 & $3,5-4,3$ \\
\hline 1995 & 10,9 & $10,1-11,6$ & 19,2 & $17,8-20,6$ & 3,3 & $2,6-3,9$ \\
\hline 1997 & 11,3 & $10,6-12,1$ & 19,6 & $18,2-21,1$ & 3,8 & $3,1-4,4$ \\
\hline 2001 & 10,0 & $9,6-10,4$ & 17,1 & $16,3-17,8$ & 3,5 & $3,1-3,8$ \\
\hline 2006 & 4,7 & $4,4-4,9$ & 7,5 & $7,1-8,0$ & 1,9 & $1,7-2,2$ \\
\hline 2009 & 2,5 & $2,3-2,7$ & 3,5 & $3,2-3,9$ & 1,4 & $1,2-1,7$ \\
\hline $2011-2012$ & 1,3 & $1,1-1,4$ & 1,9 & $1,6-2,2$ & 0,7 & $0,5-0,8$ \\
\hline
\end{tabular}

*Bebedor promedio de alto riesgo: Ha consumido alcohol en los últimos 12 meses con cualquier frecuencia y cantidad $\geq 40$ $\mathrm{g} /$ día en hombres y $\geq 24 \mathrm{~g} /$ día en mujeres. †Prevalencias estandarizadas por edad (edad promedio de la serie)

1,3\% en 2011-2012. El descenso fue rápido desde 1987 a 1993, estabilizándose durante la década de los noventa para descender de nuevo a partir del año 2000. Este descenso se produjo en ambos sexos y en todos los grupos de edad (datos de grupos de edad no mostrados en tablas).

\section{DISCUSIÓN}

Los principales resultados de este estudio muestran que en España la situación actual del consumo de alcohol en población adulta es compatible con la de los países en transición, en los que el patrón binge drinking es el que más contribuye al riesgo global derivado del consumo de alcohol, se produce una fuerte tendencia descendente del consumo promedio de riesgo y la cerveza ha desplazado al vino como la bebida preferente. Sus resultados son consistentes con estudios previos realizados en España que analizan los aspectos epidemiológicos del consumo de alcohol ${ }^{16,17}$.

Teniendo en cuenta el consumo promedio, la magnitud de la prevalencia de bebedores de alto riesgo es menor del 4,4\% estimado en EDADES $2009^{11}$ y del $5,8 \%$ del Estudio de Nutrición y Riesgo Cardiovascular en España (ENRICA) en población $\geq 18$ $\operatorname{años}^{5}$. Aunque estas diferencias disminuyen si tenemos en cuenta la tendencia descendente de este indicador, las estimaciones a través de la ENS 2011-2012 son inferiores. Respecto al binge drinking es difícil establecer comparaciones debido a las diferencias metodológicas en su medición. En el EDADES 2009 se estimó un 14,9\% de consumidores de 5 o más bebidas alcohólicas (no se utilizaron bebidas estándar) en hombres y 4 o más en mujeres en los últimos 30 días ${ }^{11}$. En el ENRICA, definiendo como el consumo en una sesión de bebida de $\geq 80 \mathrm{~g}$ en hombres y $\geq 60 \mathrm{~g}$ en mujeres en los últimos 30 días, se observó un 7,2\% de binge drinkers ${ }^{5}$.

En la mayoría de las sociedades la población adulta tiene un patrón diferenciado de consumo de alcohol según el sexo. Los hombres beben más frecuentemente y en mayores cantidades que las mujeres y como consecuencia tienen más problemas asociados al consumo de alcohol ${ }^{18}$. Aunque, al igual que ha ocurrido en el consumo de tabaco, se ha sugerido la hipótesis de convergencia, todavía las diferencias en la prevalencia entre hombres y mujeres son importantes ${ }^{19}$.

Dos enfoques teóricos se han emplazado para explicar estas diferencias. Uno de ellos enfatiza las diferencias biológicas en la 
ingesta de alcohol, basándose en el hecho de que las mujeres absorben y metabolizan el alcohol de diferente manera. Principalmente, las mujeres por su menor contenido corporal de agua para un mismo peso, tienen mayores concentraciones de alcohol en sangre después de beber cantidades equivalentes de alcohol, lo que podría influir en beber menos para obtener los mismos efectos ${ }^{20}$. Sin embargo, las explicaciones biológicas tienen muchas debilidades ya que no existen evidencias de que las mujeres limiten el consumo de bebidas alcohólicas debido a una mayor percepción de intoxicación a menores dosis que los hombres. Tampoco se han demostrado diferencias de consumo en mujeres con diferente contenido corporal de agua. No explica por qué no sólo las mujeres beben menos cantidad de alcohol sino también menos frecuentemente.

Un segundo enfoque destaca las razones culturales y sociales, desde la evidencia de que el consumo de alcohol en las mujeres ha sido sistemáticamente restringido bajo la creencia de que puede afectar adversamente el comportamiento o rol social de la mujer, considerándolo incompatible con las responsabilidades domésticas y signo de fallo del control sobre las relaciones familiares y sociales $^{20}$.

Mientras que el consumo promedio de riesgo es más prevalente en edades intermedias de la vida, el binge drinking se produce más frecuentemente en el último periodo de la adolescencia y primera etapa de la edad adulta, al igual que ocurre en otros países de nuestro entorno ${ }^{21}$. Este consumo en edades tempranas de grandes cantidades de alcohol relacionado con la búsqueda de rápidos efectos psicoactivos no era frecuente en los países mediterráneos y podría estar relacionado con la disminución de la edad de inicio del consumo de alcohol que se ha producido en España ${ }^{22}$.

Normalmente, cuando se investigan desigualdades en salud, aquellas personas que pertenecen a grupos sociales más desfavorecidos, tienen peor salud y mayor mortalidad que las personas de mayor posición socioeconómica ${ }^{23,24}$. Sin embargo, en el caso del consumo de alcohol se produce una desviación de este patrón. En los países desarrollados, las personas con mejor posición socioeconómica tienden a consumir alcohol de forma regular pero en cantidades moderadas mientras que los de posición socioeconómica más baja muestran una proporción mayor de abstención, aunque los que beben ingieren mayores cantidades y presentan más problemas relacionados con el consumo. Además, estos patrones varían entre hombres y mujeres. Según un estudio llevado a cabo en 15 países por Bloomfield et al. ${ }^{25}$, en Alemania, Holanda, Francia, Suiza, y Austria las mujeres con un elevado nivel educativo consumen más alcohol mientras que los hombres con menor nivel de estudios tienen mayor probabilidad de ser bebedores de riesgo. En mujeres no observaron diferencias respecto al binge drinking mientras que en hombres había un gradiente social de mayor riesgo en los de menor nivel educativo. En España, el patrón diferenciado por sexo coincide con los resultados de este estudio respecto al consumo promedio de riesgo. Sin embargo, el mayor gradiente de riesgo del patrón binge drinking observado tanto en mujeres como en hombres a medida que aumenta el nivel educativo discrepa con lo observado en el estudio de Bloomfeld et $\mathrm{al}^{25}$. Sin embargo, otros autores también observan un patrón similar al descrito para España, detectando un mayor riesgo de binge drinking a medida que aumenta el nivel de estudios ${ }^{26,27}$. Estas asociaciones son consistentes también con los resultados observados en otras investigaciones realizadas en España previamen$\mathrm{te}^{5,6}$.

Un aspecto relevante es que la mayoría de los binge drinkers se clasifican como bebedores de bajo riesgo respecto al consumo promedio y, de no medir este patrón, quedarían mal clasificados desde la perspectiva 
del riesgo asociado al consumo. Por ello es importante incorporar siempre este patrón en las encuestas que estimen el consumo de alcohol ${ }^{16}$.

España se encuadra entre los países en los que la cantidad de consumo per cápita y la proporción de bebedores promedio de riesgo está descendiendo ${ }^{28-30}$, mientras que la proporción de personas con un patrón binge drinking es elevada, especialmente en jóvenes ${ }^{5,6}$. Resulta llamativo el fuerte descenso del consumo promedio de riesgo, que es consistente con los resultados observados por Regidor et al. ${ }^{29}$ con datos también de la Encuesta Nacional de Salud, aunque utilizando un punto de corte más elevado para definir a los bebedores de riesgo. Al igual que en nuestro estudio, el descenso se produce en todas las categorías de edad y sexo (datos de edad y sexo no mostrados en tablas). Esta disminución se observa también en otros países del sur de Europa como Francia o Italia y ha sido atribuida al abandono del consumo de vino principalmente en las comidas $^{31}$, al igual que observamos en nuestro estudio con datos individuales en los que la cerveza ha desplazado al vino como la bebida que más contribuye a la ingesta total de alcohol.

La principal limitación de esta investigación está relacionada con la medición autodeclarada del consumo de alcohol, la cual infraestima la prevalencia, en especial para elevadas cantidades de alcohol y el binge drinking ${ }^{32,33}$. Por otro lado, no se ha podido profundizar en la descripción de la prevalencia de exbebedores ya que la formulación de las preguntas no permite diferenciar a los individuos que consumían alcohol previamente de forma regular de aquellos que eran esporádicos o probaron el alcohol de forma puntual. Tampoco se ha podido estimar la evolución del binge drinking, dado que este indicador no ha estado disponible hasta fechas recientes.
Por el contrario, entre las fortalezas de la investigación habría que destacar que se trata de una amplia muestra representativa de todas las comunidades autónomas, lo cual facilita el análisis de las estimaciones de baja prevalencia así como la de los consumidores de alto riesgo estimados a partir de la cantidad promedio de alcohol, así como su estratificación.

Como conclusión, se observa un descenso consistente del consumo promedio de alto riesgo, donde el patrón binge drinking es el que más contribuye al riesgo global derivado del consumo de alcohol. Además, la cerveza es la bebida más consumida, lo que nos acerca al perfil de países actualmente en transición. Se observan importantes diferencias sociodemográficas en la distribución del riesgo asociado al consumo promedio o al binge drinking que pueden ayudar a planificar mejor las intervenciones de prevención y control de los problemas derivados del consumo de alcohol.

\section{AGRADECIMIENTOS}

Este estudio se ha realizado en el marco del Grupo de Trabajo sobre Alcohol de la Sociedad Española de Epidemiología. Los autores agradecen la ayuda económica del Plan Nacional sobre Drogas que ha contribuido a su funcionamiento (órdenes SSI $2800 / 2012$ y $131 / 2013$ ).

\section{BIBLIOGRAFÍA}

1. Shield KD, Rylett M, Gmel G, Gmel G, KehoeChan TA, et al. Global alcohol exposure estimates by country, territory and region for 2005--a contribution to the Comparative Risk Assessment for the 2010 Global Burden of Disease Study. Addiction. 2013; 108:912-22.

2. Bloomfield K, Stockwell T, Gmel G, Rehn N. International comparisons of alcohol consumption. Alcohol Res Health. 2003; 27:95-109.

3. Room R, Makela K. Typologies of the cultural position of drinking. J Stud Alcohol. 2000; 61:47583. 
4. Rehm L, Room M, Monteiro M, Gmel G, Graham K, Rehn N et al. Alcohol use. In: Ezzati M, Lopez AD, Rodgers A, Murray CJL, editors. Comparative quantification of health risk: global and regional burden of disease attributable to selected major risk factors. Geneva: World Health Organization. 2004. 959-1109.

5. Soler-Vila H, Galan I, Valencia-Martin JL, Leon-Munoz LM, Guallar-Castillon P, et al. Binge Drinking in Spain, 2008-2010. Alcohol Clin Exp Res. 2013. En prensa.

6. Valencia-Martín JL, Galán I, Rodríguez-Artalejo F. Binge drinking in Madrid, Spain. Alcohol Clin Exp Res. 2007; 31:1723-30.

7. Plan Nacional sobre Drogas. Encuesta Estatal sobre Uso de Drogas en Enseñanzas Secundarias. Madrid: Ministerio de Sanidad, Servicios Sociales e Igualdad;2013. Disponible en: www.pnsd.msc.es/en/Categoria2/observa/pdf/ESTUDES_2010.pdf

8. Rehm J, Rehm MX, Shield KD, Gmel G, Gual A. Alcohol consumption, alcohol dependence and related harms in Spain, and the effect of treatment-based interventions on alcohol dependence. Adicciones. 2013; 25:11-8.

9. Gutierrez-Fisac JL. Indicadores de consumo de alcohol en España. Med Clin (Barc). 1995; 104:544-50.

10. Ministerio de Sanidad, Servicios Sociales e Igualdad. Encuesta Nacional de Salud 2011. Disponible en: /www.msssi.gob.es/estadEstudios/estadisticas/encuestaNacional/encuesta2011.htm

11. Plan Nacional sobre Drogas. Encuesta Domiciliaria sobre Alcohol y Drogas en España 2009. Ministerio de Sanidad, Servicios Sociales e Igualdad. Disponible en: www.pnsd.msc.es/en/Categoria2/observa/pdf/EDADES_2009.pdf

12. Ministerio de Sanidad, Servicios Sociales e Igualdad. Evaluación de la falta de respuesta en la Encuesta Nacional de Salud 2011-2012. http://www.msssi.gob.es/estadEstudios/estadisticas/encuestaNacional/encuestaNac2011/AnalisisFaltaDeRespuesta.pdf

13. Llopis JJ, Gual A, Rodríguez-Martos A. Registro del consumo de bebidas alcohólicas mediante la unidad de bebida estándar. Diferencias geográficas. Adicciones. 2000; 12:11-19

14. World Health Organization. International Guide for Monitoring Alcohol Consumption and Related Harm. Department of Mental Health and Substance Dependence. Geneva: World Health Organization; 2000. Disponible en:whqlibdoc.who.int/hq/2000/WHO MSD MSB 00.4. pdf
15. Valencia-Martín JL, Galán I, Rodríguez-Artalejo F. Alcohol and Self-Rated Health in a Mediterranean Country: The Role of Average Volume, Drinking Pattern, and Alcohol Dependence. Alcohol Clin Exp Res. 2009; 33:240-46.

16. Galán I, Álvarez J. Magnitud del problema. En: Prevención de los problemas derivados del alcohol. $1^{\mathrm{a}}$ Conferencia de prevención y promoción de la salud en la práctica clínica en España. Madrid: Ministerio de Sanidad, Servicios Sociales e Igualdad; 2008. 8-37. Disponible en: www.msssi.gob.es/profesionales/saludPublica/prevPromocion/alcohol/docs/prevencionProblemasAlcohol.pdf

17. Villalbí JR, Brugal MT. Epidemiología del consumo de alcohol y de sus consecuencias en la salud. En: Monografía sobre el alcoholismo. Pascual F, Guardia J, editores. Barcelona: Socidrogalcohol.p. 43-74. Disponible en: www.socidrogalcohol.org/index.php?option $=$ com_docman $\&$ task $=$ doc_view $\&$ gid $=113 \&$ tmpl $=$ component $\&$ format $=$ raw $\&$ Itemid $=18$

18. Anderson P, Baumberg B. Alcohol in Europe. A Public Health perspective. London: Institute of Alcohol Studies; 2006. Disponible en: http://ec.europa.eu/health/ph determinants/life style/ alcohol/documents/alcohol_europe.pdf

19. Bloomfield K, Gmel G, Neve R, Mustonen H. Investigating Gender Convergence in Alcohol Consumption in Finland, Germany, The Netherlands, and Switzerland: A Repeated Survey Analysis. Subst Abus. 2001; 22:39-53.

20. Wilsnack RW, Vogeltanz ND, Wilsnack SC, Harris TR, Ahlstrom S, Bondy S et al. Gender differences in alcohol consumption and adverse drinking consequences: cross-cultural patterns. Addiction. 2000; 95:25165 .

21. Kuntsche E, Rehm J, Gmel G. Characteristics of binge drinkers in Europe. Soc Sci Med. 2004; 59:11327.

22. Colell E, Sanchez-Niubo A, Domingo-Salvany A. Sex differences in the cumulative incidence of substance use by birth cohort. Int J Drug Policy. 2013 24:319-25.

23. Kunst AE, Bos V, Lahelma E, Bartley M, Lissau I, Regidor $\mathrm{E}$ et al. Trends in socioeconomic inequalities in self-assessed health in 10 European countries. Int $\mathrm{J}$ Epidemiol. 2005; 34:295-305.

24. Mackenbach JP, Bos V, Andersen O, Cardano M, Costa G, Harding S et al. Widening socioeconomic inequalities in mortality in six Western European countries. Int J Epidemiol. 2003; 32:830-37. 
25. Bloomfield K, Grittner U, Kramer S, Gmel G. Social inequalities in alcohol consumption and alcoholrelated problems in the study countries of the EU concerted action 'Gender, Culture and Alcohol Problems: a Multi-national Study'. Alcohol. 2006; 41(Suppl 1): 26-36.

26. Almeida-Filho N, Lessa I, Magalhaes L, Araujo MJ, Aquino E, James SA et al. Social inequality and alcohol consumption-abuse in Bahia, Brazil. Interactions of gender, ethnicity and social class. Soc Psychiatr Epidemiol. 2005; 40:214-22.

27. Serdula MK, Brewer RD, Gillespie C, Denny CH, Mokdad A. Trends in alcohol use and binge drinking, 1985-1999: results of a multi-state survey. Am J Prev Med. 2004; 26:294-8.

28. European Health for All Database (HFA-DB). World Health Organization. Regional Office for Europe. Disponible en: www.euro.who.int/en/data-and-evidence/databases/european-health-for-all-database-hfa$\mathrm{db}$

29. Regidor E, Gutierrez-Fisac JL, de los Santos Ichaso M, Fernandez E. Trends in principal cancer risk factors in Spain. Ann Oncol. 2010; 21 (Suppl 3):37-42.

30. Galan I, Rodriguez-Artalejo F, Tobias A, Gandarillas A, Zorrilla B. Vigilancia de los factores de riesgo de enfermedades no transmisibles mediante encuesta telefónica: resultados de la Comunidad de Madrid en el periodo 1995-2003. Gac Sanit. 2005; 19:193-205.

31. La Vecchia C, Bosetti C, Bertuccio P, Castro C, Pelucchi C, Negri E. Trends in alcohol consumption in Europe and their impact on major alcohol-related cancers. Eur J Cancer Prev. 2014. 2014 Jul;23(4):319-22.

32. Midanik LT. Validity of self-reported alcohol use: a literature review and assessment. Br J Addict. 1988; 83:1019-30.

33. Perrine MW, Mundt JC, Searles JS, Lester LS. Validation of daily self-reported alcohol consumption using interactive voice response (IVR) technology. J Stud Alcohol. 1995; 56:487-90. 